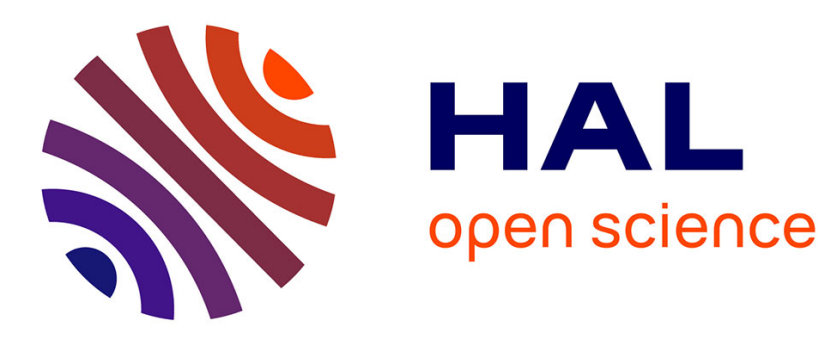

\title{
A Note on Reduced Strain Gradient Elasticity
}

\author{
Victor A. Eremeyev, Francesco Dell'Isola
}

\section{To cite this version:}

Victor A. Eremeyev, Francesco Dell'Isola. A Note on Reduced Strain Gradient Elasticity. Generalized Models and Non-classical Approaches in Complex Materials 1, pp.301-310, 2018. hal-01825523

\section{HAL Id: hal-01825523 \\ https://hal.science/hal-01825523}

Submitted on 28 Jun 2018

HAL is a multi-disciplinary open access archive for the deposit and dissemination of scientific research documents, whether they are published or not. The documents may come from teaching and research institutions in France or abroad, or from public or private research centers.
L'archive ouverte pluridisciplinaire HAL, est destinée au dépôt et à la diffusion de documents scientifiques de niveau recherche, publiés ou non, émanant des établissements d'enseignement et de recherche français ou étrangers, des laboratoires publics ou privés. 


\title{
A Note on Reduced Strain Gradient Elasticity
}

Victor A. Eremeyev and Francesco dell'Isola

\begin{abstract}
We discuss the particular class of strain-gradient elastic material models which we called the reduced or degenerated strain-gradient elasticity. For this class the strain energy density depends on functions which have different differential properties in different spatial directions. As an example of such media we consider the continual models of pantographic beam lattices and smectic and columnar liquid crystals.
\end{abstract}

\subsection{Introduction}

The Mechanics of Generalized Continua was one of the permanent interests of Prof. Gerard A. Maugin who is one of the main founder and contributor in the field, see, e.g., his recent original books and papers Maugin (2010, 2011, 2013, 2016, 2017) devoted to developments in the field. Among various generalized models of continuum the strain gradient elasticity found recently various applications in the mechanics of nano-sized solids and fluids (Forest et al, 2011; Cordero et al, 2016), for modelling of strain localization phenomena (Aifantis, 1992), as model of heterogeneous granular solids (Misra and Chang, 1993), as model of fabrics and composites (Harrison, 2016; dell'Isola and Steigmann, 2015; dell'Isola et al, 2016b; Soubestre and Boutin, 2012; d'Agostino et al, 2015; Rahali et al, 2015; Placidi et al, 2016), see also dell'Isola et al (2017); Aifantis (2014); Bertram and Glüge (2016);

\footnotetext{
Victor A. Eremeyev

Gdańsk University of Technology, ul. Gabriela Narutowicza 11/12, 80-233 Gdańsk, Poland

e-mail: eremeyev.victor@gmail.com

Francesco dell'Isola

Università di Roma "La Sapienza" \& International Research Center on Mathematics and Mechanics of Complex System (M\&MOCS), Universitá degli Studi dell'Aquila, Via Giovanni Gronchi 18 Zona industriale di Pile, 67100, L'Aquila, Italy

e-mail: francescodellisola@uniroma1.it
} 
Engelbrecht and Berezovski (2015); Chatzigeorgiou et al (2017). In this context, it is also worth mentioning continuum bodies, characterized at micro scale, by interacting spins in a crystalline lattice (see e.g. Grimmett (2016); de Masi et al $(2008,2009)$ for some relevant results using the Potts model). As some other generalized models it can predict size-effect since it includes the characteristic length observed at the nanoscale and can describe dispersion for waves propagation in microstructured solids. The typical form of the strain energy density $\mathscr{W}$ under infinitesimal deformations can be represented as follows

$$
\mathscr{W}=\mathscr{W}(e, \nabla \boldsymbol{e})
$$

or

$$
\mathscr{W}=\mathscr{W}(e, \nabla \nabla u)
$$

where

$$
\boldsymbol{e}=\frac{1}{2}\left(\nabla \boldsymbol{u}+(\nabla \boldsymbol{u})^{T}\right)
$$

is the strain tensor, $u$ is the vector of displacements, and $\nabla$ is the three-dimensional (3D) nabla-operator (Lebedev et al, 2010; Simmonds, 1994). In what follows for brevity we use form (15.2). The model was presented in the original works by Toupin (1962); Mindlin (1964); Mindlin and Eshel (1968) and in simplified form by Aifantis (1992, 2003); Askes and Aifantis (2011); Askes and Gitman (2017).

The characteristic feature of (15.1) and (15.2) is that $\mathscr{W}$ depends on a set of all second derivatives of $u$. Considering small deformations we can transform $\mathscr{W}$ into a quadratic form

$$
\mathscr{W}=\frac{1}{2} e: C: e+\frac{1}{2} \nabla \nabla u \vdots D \vdots \nabla \nabla u,
$$

where $C$ and $D$ are forth- and six-order tensors of elastic moduli, respectively. The standard assumption is that $\boldsymbol{C}$ and $D$ are not singular. Moreover, $C$ and $\boldsymbol{D}$ are assumed to be positive definite, see, e.g., Healey and Krömer (2009); Mareno and Healey (2006), that is

$$
\begin{aligned}
& \boldsymbol{e}: \boldsymbol{C}: \boldsymbol{e} \geq c \boldsymbol{e}: \boldsymbol{e} \\
& \nabla \nabla \boldsymbol{u} \vdots \boldsymbol{D} \vdots \nabla \nabla \boldsymbol{u} \geq d \nabla \nabla \boldsymbol{u} \vdots \nabla \nabla u
\end{aligned}
$$

with $c>0, d>0$. Here ":" and ":?" stand for scalar (inner) products in the spaces of second- and third-order tensors, respectively.

So, all spatial directions are equivalent in the sense of dependence on all second derivatives.

In the follows we consider another type of constitutive equations. We keep requirement (15.4) and relax (15.5) assuming that $D$ can be degenerated, that is

$$
e: C: e \geq c e: e, \quad \nabla \nabla u \vdots D: \nabla \nabla u \geq 0 .
$$


We call such model the reduced or degenerated strain gradient elasticity. In what follows we discuss few examples of such models.

\subsection{Reduced Strain Gradient Elasticity. Examples}

\subsubsection{Structural Mechanics}

First, let us recall that such inequality in spatial directions is well-known in the structural mechanics. For example, the strain energy density of an extensible beam under tension and in-plane bending is given by

$$
\mathscr{W}=\frac{1}{2} \mathbb{K}_{e}\left(u^{\prime}\right)^{2}+\mathbb{K}_{b}\left(w^{\prime \prime}\right)^{2},
$$

where $\mathbb{K}_{e}>0$ and $\mathbb{K}_{b}>0$ are the extensional and bending stiffness parameters, $u=u(x)$ and $w=w(x)$ are longitudinal and transverse displacements, respectively, $x$ is the coordinate along the beam axis, and the prime stands for derivative with respect to $x$.

The same situation can be observed in the Kirchhof-Love theory of plates and shells (Timoshenko and Woinowsky-Krieger, 1985; Lebedev et al, 2010). For example, the strain energy density of an elastic homogeneous plate of thickness $h$ is given by

$$
\begin{aligned}
\mathscr{W}= & \frac{E h}{2(1+v)}\left[u_{\alpha, \beta} u_{\alpha, \beta}+\frac{v}{1-v}\left(u_{\alpha, \alpha}\right)^{2}\right] \\
& +\frac{E h^{3}}{24(1+v)}\left[w_{, \alpha \beta} w_{, \alpha \beta}+\frac{v}{1-v}\left(w_{, \alpha \alpha}\right)^{2}\right],
\end{aligned}
$$

where $E$ and $v$ are Young's modulus and Poisson's ratio, respectively, $u_{\alpha}$ and $w$ are the in-plane and transverse displacements, $x_{\alpha}$ is a Cartesian coordinates, $\alpha=1,2$. Hereinafter indices after comma denote derivatives with respect to $x_{\alpha}$, so $w_{, \alpha}$ is the partial derivative of $w$ with respect to $x_{\alpha}$,

$$
w_{, \alpha}=\frac{\partial w}{\partial x_{\alpha}} .
$$

So, considering beams, plates and shells as 1D and 2D continua embedded into 3D physical space we see a difference in order of used derivatives in different directions. Obviously, for beams the preferable direction is tangent to the beam axis whereas for plates and shells it is normal to the middle surface. Nevertheless, here the mentioned difference concerns different components of displacements. For example, (15.8) includes all derivatives of the deflection $w$. In what follows we demonstrate another situation. 


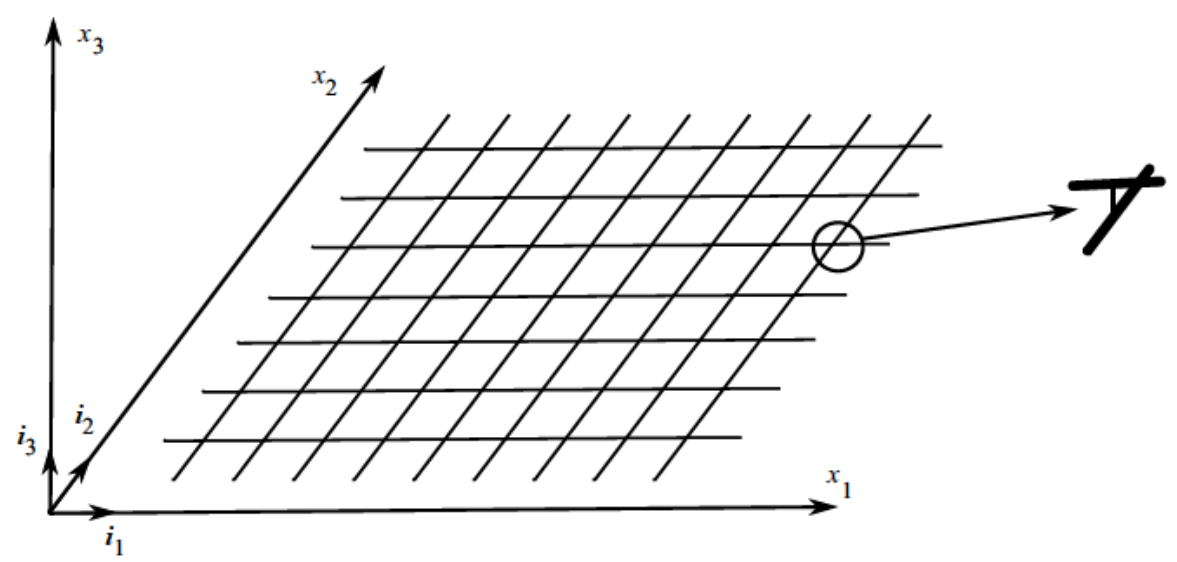

Fig. 15.1: Structure of a pantographic beam lattice.

\subsubsection{Continual Models for Pantographic Beam Lattices}

Recently, a new class of composites materials called pantographic beam lattices was introduced, see dell'Isola and Steigmann (2015); dell'Isola et al (2016a); Giorgio et al (2017); Placidi et al (2016). A scheme of a pantographic lattice is given in Fig. 15.1. It consists of two families of orthogonal long flexible beams connected by pivots. For in-plane deformations using heuristic homogenization the model of a pantographic lattice is reduced to the two-dimensional strain gradient continuum. Introducing the vector displacements as follows

$$
\boldsymbol{u}=u_{1} i_{1}+u_{2} i_{2}, \quad u_{\alpha}=u_{\alpha}\left(x_{1}, x_{2}\right),
$$

we obtain the strain energy density in the form, see dell'Isola et al (2016a); Boutin et al (2017); Placidi et al (2017) for details,

$$
\mathscr{W}=\frac{\mathbb{K}_{e}^{(1)}}{2} u_{1,1}^{2}+\frac{\mathbb{K}_{e}^{(2)}}{2} u_{2,2}^{2}+\frac{\mathbb{K}_{p}}{2}\left(u_{1,2}+u_{2,1}\right)^{2}+\frac{\mathbb{K}_{b}^{(2)}}{2} u_{1,22}^{2}+\frac{\mathbb{K}_{b}^{(1)}}{2} u_{2,11}^{2},
$$

where the stiffness parameters $\mathbb{K}_{e}^{(\alpha)}>0$ and $\mathbb{K}_{b}^{(\alpha)}>0$ are related to the extensional and bending stiffnesses of the beams at the interpivot scale, respectively, while the coefficient $\mathbb{K}_{p} \geq 0$ relates to shear stiffness of the pivots. Here we also neglect torsional deformations of the beams. In particular, if the pivots are perfect then $\mathbb{K}_{p}$ vanishes and the strain energy transforms to

$$
\mathscr{W}=\frac{\mathbb{K}_{e}^{(1)}}{2} u_{1,1}^{2}+\frac{\mathbb{K}_{e}^{(2)}}{2} u_{2,2}^{2}+\frac{\mathbb{K}_{b}^{(2)}}{2} u_{1,22}^{2}+\frac{\mathbb{K}_{b}^{(1)}}{2} u_{2,11}^{2} .
$$


Both energies are significantly degenerated since (15.9) and (15.10) include only few second derivatives. In fact, there are only $u_{2,11}$ and $u_{1,22}$. As it was shown in (dell'Isola et al, 2016a; Eremeyev et al, 2017) for (15.10) there exist nontrivial static solutions with zero energy which are different from rigid body motions.

Neglecting the torsional deformations of the beams and pivots we may also write the strain energy density for a pantographic beam lattice for three-dimensional deformations. Here $u=u_{k}\left(x_{1}, x_{2}, x_{3}\right) i_{k}, k=1,2,3$, and vectors $i_{1}$ and $i_{2}$ are directed along the corresponding beams. Under certain assumptions on the beam crosssections we obtain that

$$
\mathscr{W}=\frac{\mathbb{K}_{e}^{(1)}}{2} u_{1,1}^{2}+\frac{\mathbb{K}_{e}^{(2)}}{2} u_{2,2}^{2}+\frac{\mathbb{K}_{b}^{(2)}}{2}\left(u_{1,22}^{2}+u_{3,22}^{2}\right)+\frac{\mathbb{K}_{b}^{(1)}}{2}\left(u_{2,11}^{2}+u_{3,11}^{2}\right) .
$$

The form (15.11) corresponds to the perfect pivots that is without stored energy as in (15.10). In other words this 2D material inherits the properties of extensible beams, so its strain energy density is a "sum" of energies of beams undergoing bending and stretching deformations without torsion.

The advantages in 3D printing gives us also the possibility to produce threedimensional pantographic beam lattices such as shown in Fig. 15.2. Neglecting the energies of beams due to torsion as well as energies due to torsion and bending in pivots we introduce the extension of the 2D model (15.11) to the 3D case as follows

$$
\begin{aligned}
\mathscr{W}= & \frac{\mathbb{K}_{e}^{(1)}}{2} u_{1,1}^{2}+\frac{\mathbb{K}_{e}^{(2)}}{2} u_{2,2}^{2}+\frac{\mathbb{K}_{b}^{(2)}}{2}\left(u_{1,22}^{2}+u_{3,22}^{2}\right)+\frac{\mathbb{K}_{b}^{(1)}}{2}\left(u_{2,11}^{2}+u_{3,11}^{2}\right) \\
& +\frac{\mathbb{K}_{e}^{(3)}}{2} u_{3,3}^{2} .
\end{aligned}
$$

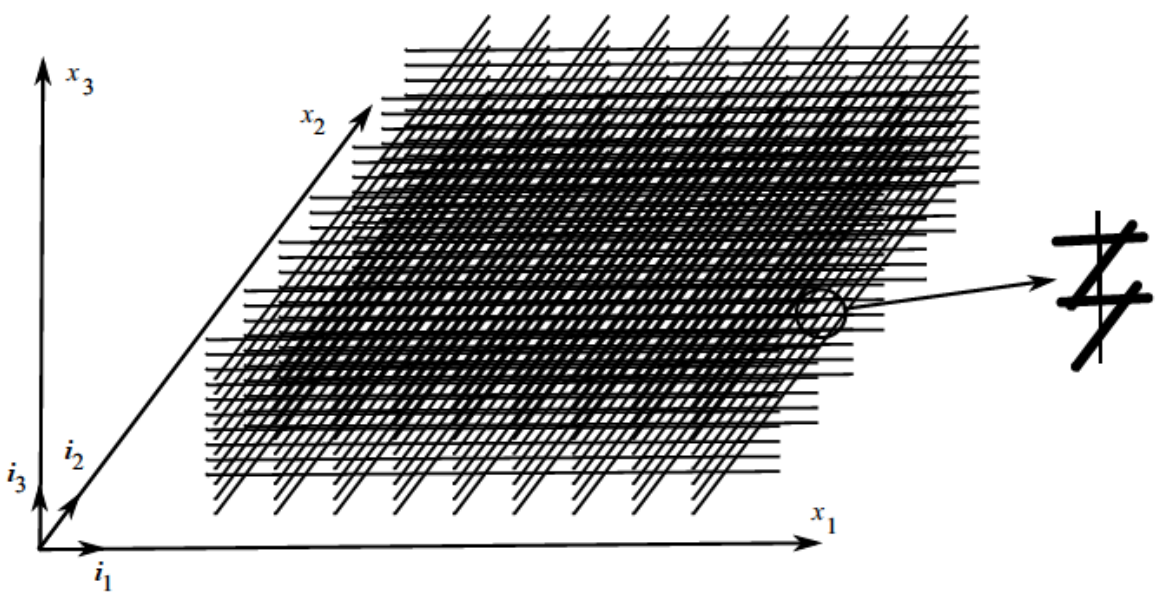

Fig. 15.2: Three-dimensional pantographic beam lattice. 
Here $\mathbb{K}_{e}^{(3)}$ is the stiffness related to pivots undergoing tension/compression.

Comparing (15.12) with the general constitutive equation (15.3) we see that $\boldsymbol{D}$ is rather degenerated. Thus, the presented above models belong to the class of constitutive relations of reduced strain gradient elasticity. The pantographic beam structures are not unique example of such materials, other examples one can find in the hydrostatics of liquid crystals.

\subsubsection{Smectics and Columnar Liquid Crystals}

Smectics are the particular class of liquid crystals with layered structure, see Chandrasekhar (1977); de Gennes and Prost (1993); Oswald and Pieranski (2006). Each layer is a two-dimensional liquid. Nevertheless smectics demonstrate existence of non-hydrostatic stresses and resistance to bending. The schematic structure of smectic A is given in Fig. 15.3 (a) where ordered arrangements of molecules in each layer is shown. For small distortion of the initially plane layers of a smectic the main kinematical descriptor is the displacement field $\boldsymbol{u}=u(x, y, z) \boldsymbol{i}_{3}$. So, the kinematics of smectics is determined by one scalar function $u$.

The strain energy density of smectics A is given

$$
\mathscr{W}=\frac{1}{2} \mathbb{B}\left(u_{, z}\right)^{2}+\frac{1}{2} \mathbb{K}\left(u_{, x x}+u_{, y y}\right)^{2},
$$

where $\mathbb{B}$ and $\mathbb{K}$ are elastic moduli describing the longitudinal stiffness along $z$ direction and the bending stiffness of the smectic layers, respectively.

Another type of liquid crystals are so-called columnar phases which structure is shown in Fig. 15.3 (b). From the physical point of view these liquid crystals look as set of flexible deformable tubes which can easily slide along each other. For small deformations of this class of liquid crystals we introduce the vector of displacements

|||||||||||||||| ||||||||||||||||

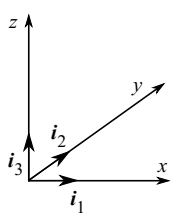
||||||||||||||||

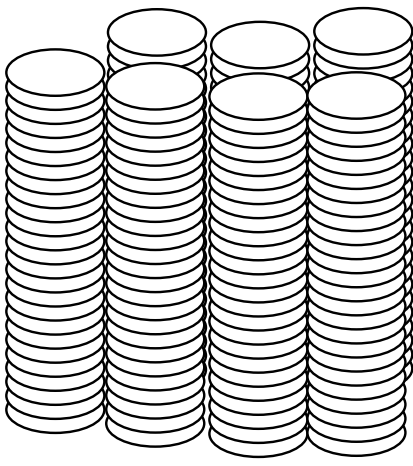

b)

Fig. 15.3: Structures of liquid crystals. a) smectic A, b) a columnar. 
$\boldsymbol{u}=u_{1} \boldsymbol{i}_{1}+u_{2} \boldsymbol{i}_{2}+u_{3} \boldsymbol{i}_{3}, u_{k}=u_{k}(x, y, z)$. The strain energy density is now given by de Gennes and Prost (1993) as follows

$$
\begin{aligned}
\mathscr{W}= & \frac{1}{2} \mathbb{B}\left(u_{1, x}+u_{2, y}\right)^{2}+\frac{1}{2} \mathbb{K}_{1}\left[\left(u_{1, x}-u_{2, y}\right)^{2}+\left(u_{1, y}+u_{2, x}\right)^{2}\right] \\
& +\frac{1}{2} \mathbb{K}_{2}\left[u_{1, z z}^{2}+u_{2, z z}^{2}\right],
\end{aligned}
$$

where $\mathbb{B}, \mathbb{K}_{1}$ and $\mathbb{K}_{2}$ are elastic moduli.

The similarity between strain energies (15.13) and (15.14) of these liquid crystals and the form of strain energy densities of pantographic beam lattices (15.12) is obvious. Indeed, all these forms contains incomplete set of second spatial derivatives, so they belong to the class of materials described using the reduced strain gradient elasticity.

\subsubsection{Other Spatially Non-Symmetric Models}

Almost all models known in structural and continuum mechanics are symmetric in spatial directions in mentioned above sense. Nevertheless, non-symmetric models are also known. Let us mention few equations of such kind.

The Kadomtsev-Petwiashvili equation is a two-dimensional analog of the famous Korteweg-de-Vries equation, see Kadomtsev and Petviashvili (1970). It takes the form

$$
\left(u_{, t}+6 u u_{, x}+u_{, x x x}\right)_{, x}+3 \lambda u_{, y y}=0,
$$

where $u=u(x, y, t), x$ and $y$ are Cartesian coordinates and $\lambda= \pm 1$. Eq. (15.15) cames originally from acoustics and found further applications in the nonlinear theory of dispersive waves, theory of solitons, theory of ferromagnetics, string theory, see, e.g., Ablowitz and Segur (1981); Ablowitz and Clarkson (1991); Maugin (1999). The Kadomtsev-Petviashvili equation describes also the evolution of the localized structures over a large-time scale of a quasi-continuum model deduced from a nonlinear lattice model, see Pouget (2005).

The next example is the PDE arose in the analysis of gas centrifuges, nowadays known as Onsager's pancake equation (Wood and Morton, 1980; Eastham and Peterson, 2004). It is given by

$$
\left[e^{x}\left(e^{x} u_{, x x}\right)_{, x x}\right]_{, x x}+b u_{, y y}=f(x, y),
$$

where $u=u(x, y), b$ and $f$ are given.

Both equations (15.15) and (15.16) were derived using some approximation techniques in which the preferable spatial direction was chosen a priori. 


\subsection{Conclusions}

We briefly discussed a new class of strain gradient elasticity models called the reduced or degenerated strain gradient elasticity. Among such continua there are continual models of pantographic beam lattices and smectic and columnar liquid crystals. The common peculiarity for both media is a layered structure and sensitivity to bending only in certain directions. From the mathematical point of view the corresponding systems of partial differential equations and natural boundary conditions requires special analysis, such as given in Eremeyev et al (2017) within the framework of anisotropic Sobolev spaces. Let us note that the considered model is obviously anisotropic with a special type of anisotropy. Usually, the anisotropic behavior of solids is determines by symmetries of the tensors of elastic moduli. Here, in addition to this symmetries we meet strong dependence of the material properties on the spatial directions since the strain energy density may be independent on some components of strain gradient tensor. In particular, for pantographic beam lattice as for smectic and columnar liquid crystals there is a preferable direction normal to the inner layers. Here we are restricted ourselves by infinitesimal deformations, but the models can be easily extended for finite deformations. So, we can easily consider the reduced strain gradient elasticity for large deformations. Another common peculiarity observed for pantographic beam lattices and liquid crystals is the existence of non-trivial solutions with zero energy. The further analysis of such constitutive equations can be performed using the material symmetry group defined in (Bertram, 2016) as was performed for shells in (Eremeyev and Pietraszkiewicz, 2006) and for micropolar media in (Eremeyev and Pietraszkiewicz, 2012, 2016).

\section{References}

Ablowitz MA, Clarkson PA (1991) Solitons, Nonlinear Evolution Equations and Inverse Scattering, London Mathematical Society lecture note series, vol 149. Cambridge University Press, Cambridge

Ablowitz MJ, Segur H (1981) Solitons and the inverse scattering transform. SIAM, Philadelphia

Aifantis EC (1992) On the role of gradients in the localization of deformation and fracture. Int J Engng Sci 30(10):1279 - 1299

Aifantis EC (2003) Update on a class of gradient theories. Mech Materials 35(3):259-280

Aifantis EC (2014) Gradient material mechanics: perspectives and prospects. Acta Mech 225(45):999-1012

Askes H, Aifantis EC (2011) Gradient elasticity in statics and dynamics: An overview of formulations, length scale identification procedures, finite element implementations and new results. Int J Solids Struct 48(13):1962-1990

Askes H, Gitman I (2017) Reducible and irreducible forms of stabilised gradient elasticity in dynamics. Math Mech Complex Systems 5(1):1-17

Bertram A (2016) Compendium on Gradient Materials . OvGU, Magdeburg

Bertram A, Glüge R (2016) Gradient materials with internal constraints. Math Mech Complex Systems 4(1):1-15

Boutin C, dell'Isola F, Giorgio I, Placidi L (2017) Linear pantographic sheets: Asymptotic micromacro models identification. Math Mech Complex Systems 5(2):127-162 
Chandrasekhar S (1977) Liquid Crystals. Cambridge University Press, Cambridge, UK

Chatzigeorgiou G, Meraghni F, Javili A (2017) Generalized interfacial energy and size effects in composites. J Mech Phys Solids 106:257-282

Cordero NM, Forest S, Busso EP (2016) Second strain gradient elasticity of nano-objects. J Mech Phys Solids 97:92-124

d'Agostino MV, Giorgio I, Greco L, Madeo A, Boisse P (2015) Continuum and discrete models for structures including (quasi-) inextensible elasticae with a view to the design and modeling of composite reinforcements. Int J Solids Struct 59:1-17

dell'Isola F, Steigmann D (2015) A two-dimensional gradient-elasticity theory for woven fabrics. J Elast 118(1):113-125

dell'Isola F, Giorgio I, Pawlikowski M, Rizzi N (2016a) Large deformations of planar extensible beams and pantographic lattices: Heuristic homogenisation, experimental and numerical examples of equilibrium. Proc Roy Soc London A 472(2185):20150,790

dell'Isola F, Steigmann D, della Corte A (2016b) Synthesis of fibrous complex structures: Designing microstructure to deliver targeted macroscale response. Appl Mech Rev 67(6):060,804-060,80421

dell'Isola F, Della Corte A, Giorgio I (2017) Higher-gradient continua: The legacy of Piola, Mindlin, Sedov and Toupin and some future research perspectives. Math Mech Solids 22(4):852-872

Eastham JF, Peterson JS (2004) The finite element method in anisotropic Sobolev spaces. Computers \& Mathematics with Applications 47(10):1775-1786

Engelbrecht J, Berezovski A (2015) Reflections on mathematical models of deformation waves in elastic microstructured solids. Math Mech Complex Systems 3(1):43-82

Eremeyev VA, Pietraszkiewicz W (2006) Local symmetry group in the general theory of elastic shells. J Elast 85(2):125-152

Eremeyev VA, Pietraszkiewicz W (2012) Material symmetry group of the non-linear polar-elastic continuum. Int J Solids Struct 49(14):1993-2005

Eremeyev VA, Pietraszkiewicz W (2016) Material symmetry group and constitutive equations of micropolar anisotropic elastic solids. Math Mech Solids 21(2):210-221

Eremeyev VA, dell'Isola F, Boutin C, Steigmann D (2017) Linear pantographic sheets: existence and uniqueness of weak solutions. J Elast DOI 10.1007/s10659-017-9660-3

Forest S, Cordero N, Busso EP (2011) First vs. second gradient of strain theory for capillarity effects in an elastic fluid at small length scales. Comput Materials Sci 50(4):1299-1304

de Gennes G P, Prost J (1993) The Physics of Liquid Crystals, 2nd edn. Clarendon Press, Oxford

Giorgio I, Rizzi N, Turco E (2017) Continuum modelling of pantographic sheets for outof-plane bifurcation and vibrational analysis. Proc Roy Soc A 473(2207):21 pages DOI 10.1098/rspa.2017.0636

Grimmett G (2016) Correlation inequalities for the Potts model. Math Mech Complex Systems 4(3):327-334

Harrison P (2016) Modelling the forming mechanics of engineering fabrics using a mutually constrained pantographic beam and membrane mesh. Composites A 81:145-157

Healey TJ, Krömer S (2009) Injective weak solutions in second-gradient nonlinear elasticity. ESAIM: Control, Optimisation and Calculus of Variations 15(4):863-871

Kadomtsev BB, Petviashvili VI (1970) On the stability of solitary waves in weakly dispersing media. Sov Phys Doklady 15(6):539-541

Lebedev LP, Cloud MJ, Eremeyev VA (2010) Tensor Analysis with Applications in Mechanics. World Scientific, New Jersey

Mareno A, Healey TJ (2006) Global continuation in second-gradient nonlinear elasticity. SIAM J Math Analysis 38(1):103-115

de Masi A, Merola I, Presutti E, Vignaud Y (2008) Potts models in the continuum. uniqueness and exponential decay in the restricted ensembles. J Stat Phys 133(2):281-345

de Masi A, Merola I, Presutti E, Vignaud Y (2009) Coexistence of ordered and disordered phases in Potts models in the continuum. J Stat Phys 134(2):243-306

Maugin GA (1999) Nonlinear Waves in Elastic Crystals. Oxford University Press, Oxford 
Maugin GA (2010) Generalized continuum mechanics: what do we mean by that? In: Maugin GA, Metrikine AV (eds) Mechanics of Generalized Continua. One Hundred Years after the Cosserats, Springer, pp 3-13

Maugin GA (2011) A historical perspective of generalized continuum mechanics. In: Altenbach H, Erofeev VI, Maugin GA (eds) Mechanics of Generalized Continua. From the Micromechanical Basics to Engineering Applications, Springer, Berlin, pp 3-19

Maugin GA (2013) Generalized Continuum Mechanics: Various Paths, Springer, Dordrecht, pp 223-241

Maugin GA (2016) Continuum Mechanics Through Ages. From the Renaissance to the Twentieth Century. Springer, Cham

Maugin GA (2017) Non-Classical Continuum Mechanics: A Dictionary. Springer, Singapore

Mindlin RD (1964) Micro-structure in linear elasticity. Arch Ration Mech Analysis 16(1):51-78

Mindlin RD, Eshel NN (1968) On first strain-gradient theories in linear elasticity. Int J Solids Struct 4(1):109-124

Misra A, Chang CS (1993) Effective elastic moduli of heterogeneous granular solids. Int J Solids Struct 30:2547-2566

Oswald P, Pieranski P (2006) Smectic and Columnar Liquid Crystals: Concepts and Physical Properties Illustrated by Experiments. The Liquid Crystals Book Series (eds GW Gray, JW Goodby, and A Fukuda), Taylor \& Francis, Boca Raton

Placidi L, Barchiesi E, Turco E, Rizzi NL (2016) A review on 2D models for the description of pantographic fabrics. ZAMP 67(5):121

Placidi L, Andreaus U, Giorgio I (2017) Identification of two-dimensional pantographic structure via a linear D4 orthotropic second gradient elastic model. J Engng Math 103(1):1-21

Pouget J (2005) Non-linear lattice models: complex dynamics, pattern formation and aspects of chaos. Phil Magazine 85(33-35):4067-4094

Rahali Y, Giorgio I, Ganghoffer JF, dell'Isola F (2015) Homogenization à la Piola produces second gradient continuum models for linear pantographic lattices. Int J Engng Sci 97:148-172

Simmonds JG (1994) A Brief on Tensor Analysis, 2nd edn. Springer, New Yourk

Soubestre J, Boutin C (2012) Non-local dynamic behavior of linear fiber reinforced materials. Mech Materials 55:16-32

Timoshenko SP, Woinowsky-Krieger S (1985) Theory of Plates and Shells. McGraw Hill, New York Toupin RA (1962) Elastic materials with couple-stresses. Arch Ration Mech Analysis 11(1):385-414 Wood HG, Morton JB (1980) Onsager's pancake approximation for the fluid dynamics of a gas centrifuge. J Fluid Mech 101(1):1-31 